Supporting Information

\title{
Oligomer Electrolytes for Light-Emitting Electrochemical Cells: Influence of the End-Groups on Ion Coordination, Ion Binding and Turn-on Kinetics
}

Isabelle Gerz ${ }^{1}$, E. Mattias Lindh ${ }^{2}$, Pall Thordarson ${ }^{3}$, Ludvig Edman ${ }^{2}$, Jolla Kullgren ${ }^{1}$, Jonas Mindemark ${ }^{1, *}$

${ }^{1}$ Department of Chemistry - Ångström Laboratory, Uppsala University, Box 538, SE-751 21 Uppsala, Sweden

2 The Organic Photonics and Electronics Group, Department of Physics, Umeå University, SE-901 87 Umeå, Sweden

${ }^{3}$ School of Chemistry, the Australian Centre for Nanomedicine and the ARC Centre of Excellence in Convergent Bio-Nano Science \& Technology, University of New South Wales, Sydney, NSW 2052, Australia

* Corresponding author. E-mail: jonas.mindemark@kemi.uu.se 


\section{Supramolecular fits}

In the paper, two different binding models are considered (see previous work for full details on the equations and terminology used here).

1:1 equilibria. Here, we define the ${ }^{7} \mathrm{Li}$ NMR resonance for the host $\left(\mathrm{Li}^{+}\right)$as $\delta_{\mathrm{H}}$ and the hostguest complex as $\delta_{\text {HG. }}$. From this, we can also define the change in resonance for the hostguest complexation as $\delta_{\Delta \mathrm{HG}}=\delta_{\mathrm{HG}}-\delta_{\mathrm{H}}$. If we then define $\delta_{0}=\mathrm{NMR}$ resonance of the host before the guest is added (before the start of titration), we can define the change in chemical shift as $\Delta \delta=\delta-\delta_{0}$. We can now write the NMR version of our simple 1:1 equilibria according to Equation (S1) which is derived from the generic quadratic equation used to calculate the concentration of host-guest complex [HG] as previously described. ${ }^{1,2}$

$$
\Delta \delta=\frac{\delta_{\Delta \mathrm{HG}}}{[\mathrm{H}]_{0}}\left(\frac{1}{2}\left\{\left([\mathrm{G}]_{0}+[\mathrm{H}]_{0}+\frac{1}{K_{\mathrm{a}}}\right)-\sqrt{\left([\mathrm{G}]_{0}+[\mathrm{H}]_{0}+\frac{1}{K_{\mathrm{a}}}\right)^{2}+4[\mathrm{H}]_{0}[\mathrm{G}]_{0}}\right\}\right)
$$

1:2 model. In this paper, we use the additive model "flavor". ${ }^{2}$ This means that we make the assumption that that the chemical shift changes are simply additive: $\delta_{\Delta \mathrm{HG}_{2}}=2 \delta_{\Delta \mathrm{HG}}$. This yields equation $(\mathrm{S} 2)^{2}$

$$
\Delta \delta=\frac{\delta_{\Delta \mathrm{HG}} K_{1}[\mathrm{G}]\left(1+2 K_{2}[\mathrm{G}]\right)}{1+K_{1}[\mathrm{G}]+K_{1} K_{2}[\mathrm{G}]^{2}}
$$

The raw input data, the calculated fit, statistical information and associated information can be accessed via the www.supramolecular.org database through the below unique URLs:

\section{TMPE-OH}

http://app.supramolecular.org/bindfit/view/8496ec25-a7ae-4482-b6cf-f6403441ca4a

\section{$\mathrm{TMPE}-\mathrm{OCH}_{3}$}

http://app.supramolecular.org/bindfit/view/29d16ec9-1049-4b60-b42c-eddb23deae9d

TMPE-EC

http://app.supramolecular.org/bindfit/view/4a6f20a2-57fc-44f6-b7c1-2d0f888f0e8d

$T M P E-B C$

http://app.supramolecular.org/bindfit/view/b48972b1-144a-44bb-b3c3-3ab768f5c517

TMPE-OC

http://app.supramolecular.org/bindfit/view/6a6ac2bf-03ac-4d6a-a59d-58f71015a185 


\section{Calculation of inter-complex distances}

We first consider the concentrated bulk electrolyte with a 1:1 ion transporter $: \mathrm{LiCF}_{3} \mathrm{SO}_{3}$ ratio. In each volume $V$ of bulk electrolyte, there will be $N=\frac{V \cdot \rho_{\mathrm{el}} N_{\mathrm{A}}}{M_{\mathrm{el}}}$ electrolyte complexes. In order to estimate the average nearest-neighbor distance between complexes, we can treat each ion transporter: $\mathrm{LiCF}_{3} \mathrm{SO}_{3}$ complex as being located at the center of an imaginary sphere and calculating the radius of this sphere in analogy with the Wigner-Seitz radius used in condensed matter physics. As illustrated in Figure 6, the inter-complex distance is $2 r_{\mathrm{s}}$, where $r_{\mathrm{s}}$ is the sphere radius given by

$$
r_{\mathrm{s}}=\sqrt[3]{\frac{3}{4 \pi} \cdot \frac{V_{\mathrm{s}}}{N}}
$$

and $V_{\mathrm{s}}$ is the total sphere volume. The most efficient arrangement of such spheres (that results in the lowest volume) is a closest-packed configuration (see Figure 6a), in which the spheres occupy $\frac{\pi}{3 \sqrt{2}}(\approx 74 \%)$ of the total volume $V$. The average volume per electrolyte sphere is thus given by

$$
\frac{V_{\mathrm{s}}}{N}=\frac{\pi}{3 \sqrt{2}} \cdot \frac{M_{\mathrm{el}}}{\rho_{\mathrm{el}} \cdot N_{\mathrm{A}}}
$$

Combining these equations and accounting for electrolyte dilution (in the active material of an LEC) to a volume fraction $\phi_{\mathrm{el}}$ leads to the following equation for $r_{\mathrm{s}}$ :

$$
r_{\mathrm{s}}=\sqrt[3]{\frac{1}{4 \sqrt{2}} \cdot \frac{M_{\mathrm{el}}}{\phi_{\mathrm{el}} \cdot \rho_{\mathrm{el}} \cdot N_{\mathrm{A}}}}
$$

For TMPE- $\mathrm{OCH}_{3}: \mathrm{LiCF}_{3} \mathrm{SO}_{3}$, which has a density $\rho_{\mathrm{el}}=1.1 \mathrm{~g} \mathrm{~cm}^{-3}$ (estimated gravimetrically) and an approximate molar mass $M_{\mathrm{el}}=648 \mathrm{~g} \mathrm{~mol}^{-1}$, the distance between two nearest-neighbor complexes in the bulk electrolyte is given by $2 r_{\mathrm{s}}=1.1 \mathrm{~nm}$.

In the active material of an LEC, the electrolyte is effectively diluted by the conjugated polymer (e.g., SY). Assuming additive volumes, we can convert the weight ratio of 1:0.11:0.03 (SY:TMPE-OCH $\mathrm{LiCF}_{3} \mathrm{SO}_{3}$ ) in a typical active material (the density of $\mathrm{SY}$ has been approximated to $\left.1.0 \mathrm{~g} \mathrm{~cm}^{-3}\right)^{3}$ to a volume fraction $\phi_{\mathrm{el}}=0.11$. This gives an average distance between complexes $2 r_{\mathrm{s}}=2.3 \mathrm{~nm}$ in the active material blend. 


\section{Supporting figures}
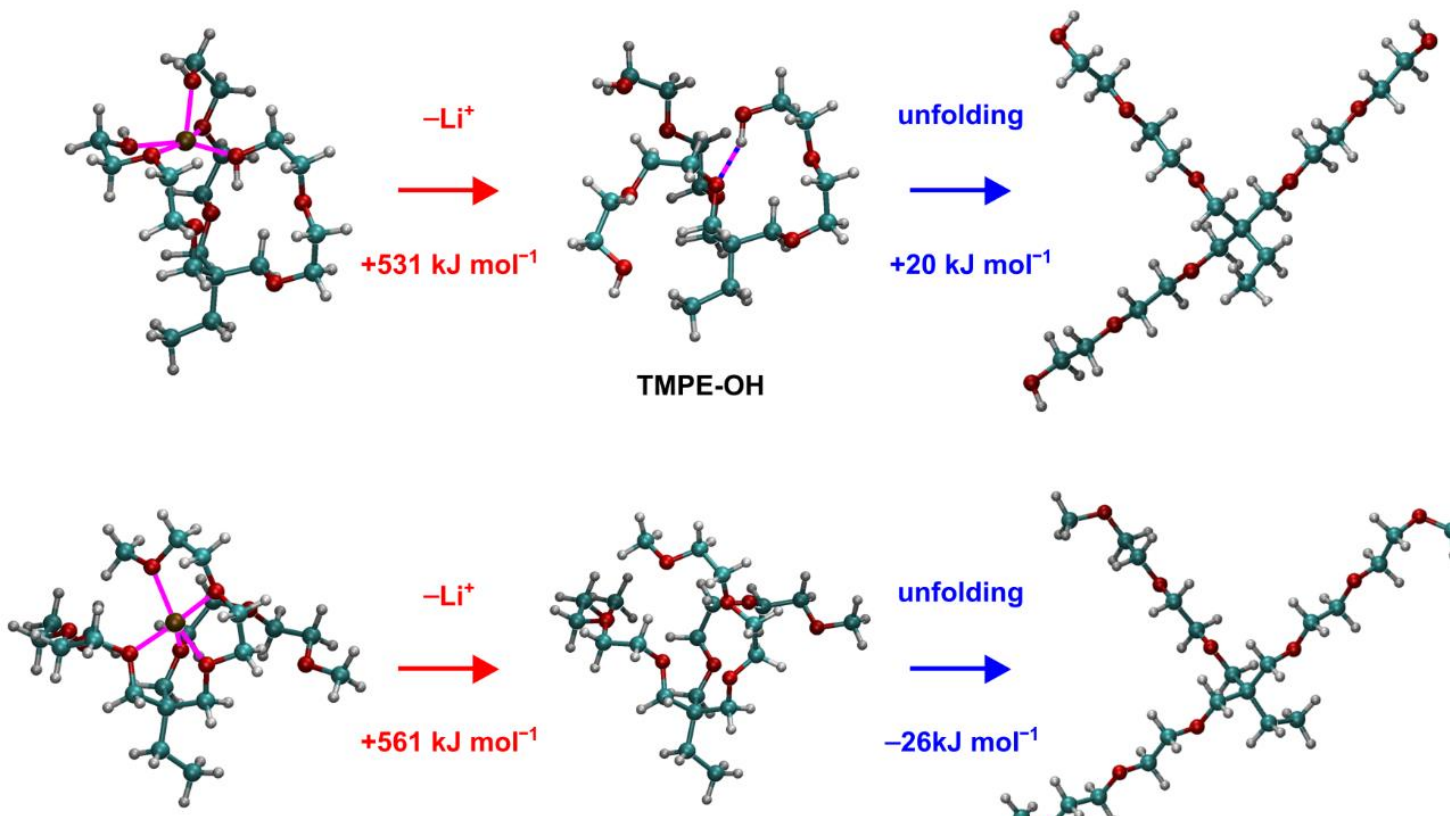

TMPE-OCH


Figure S1. Schematic of the procedure for calculating the instantaneous binding energy (red arrows) as well as the unfolded binding energy (blue arrows) for the most energetically favorable conformations of TMPE-OH (top), TMPE-OCH 3 (middle) and TMPE-EC (bottom) together with the respective energy differences for the different steps. The binding motif has been highlighted (left column) as well as the stabilizing hydrogen bond in TMPE-OH. 
TMPE-OH

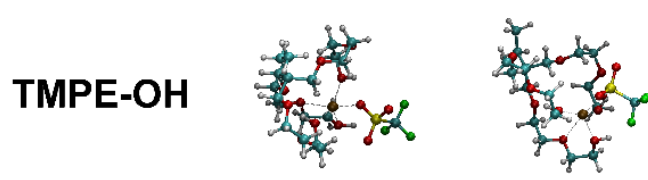

然

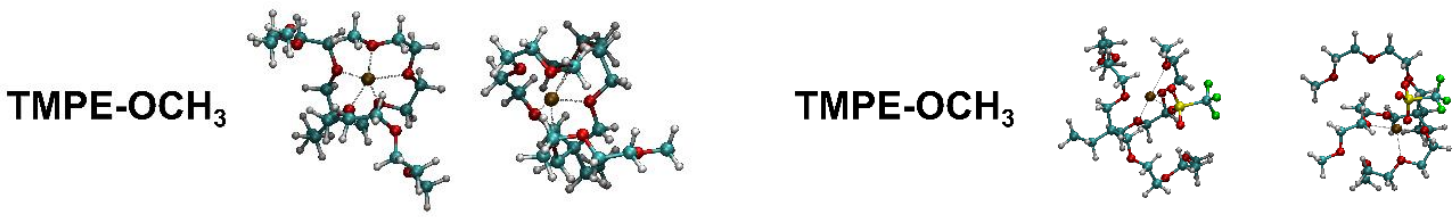

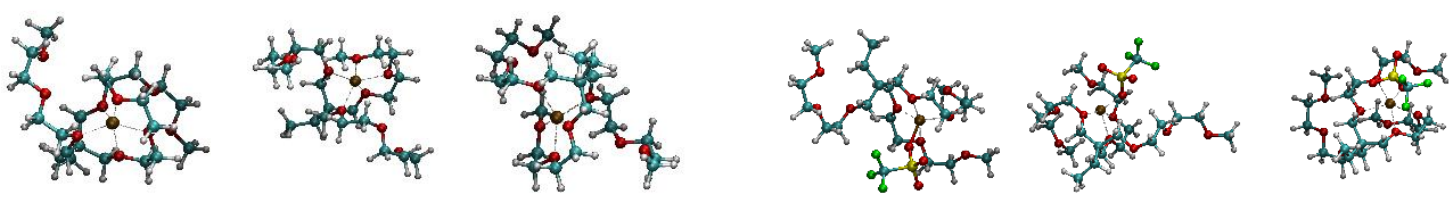

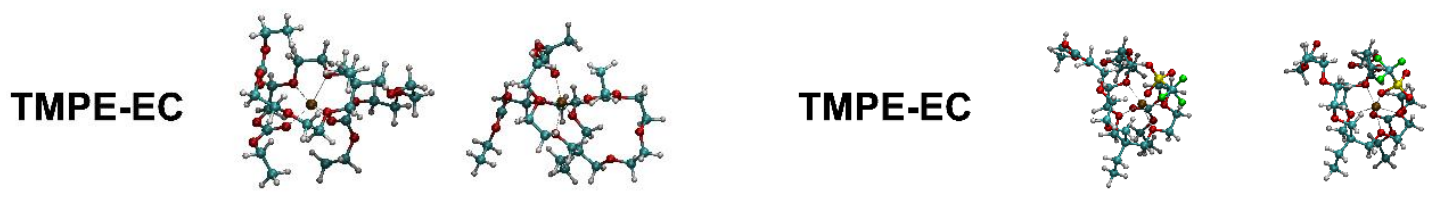

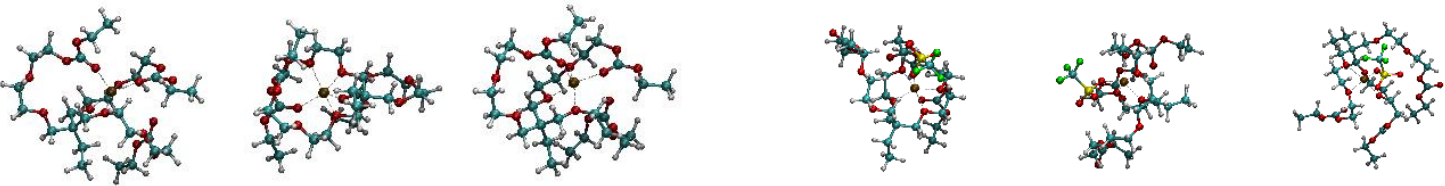

TMPE-BC

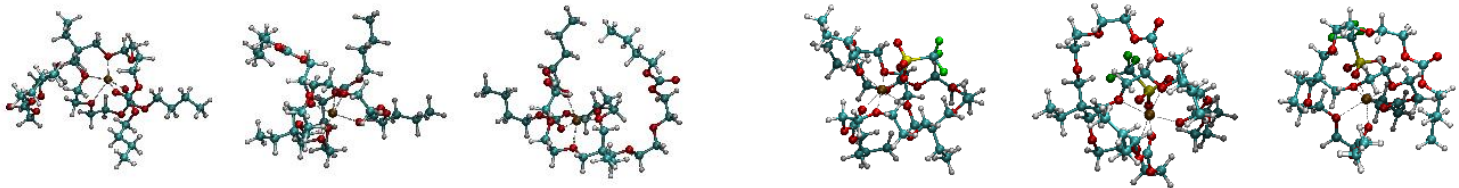

TMPE-OC

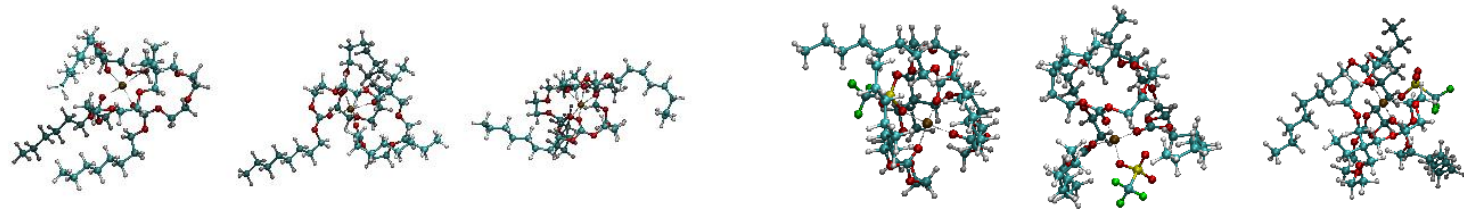


Figure S2. DFT-optimized geometries from minimum energy structures of the MD simulation for ion transporter $+\mathrm{Li}^{+}$complexes (left) and ion transporter $+\mathrm{Li}^{+}+\mathrm{CF}_{3} \mathrm{SO}_{3}{ }^{-}$ (right).
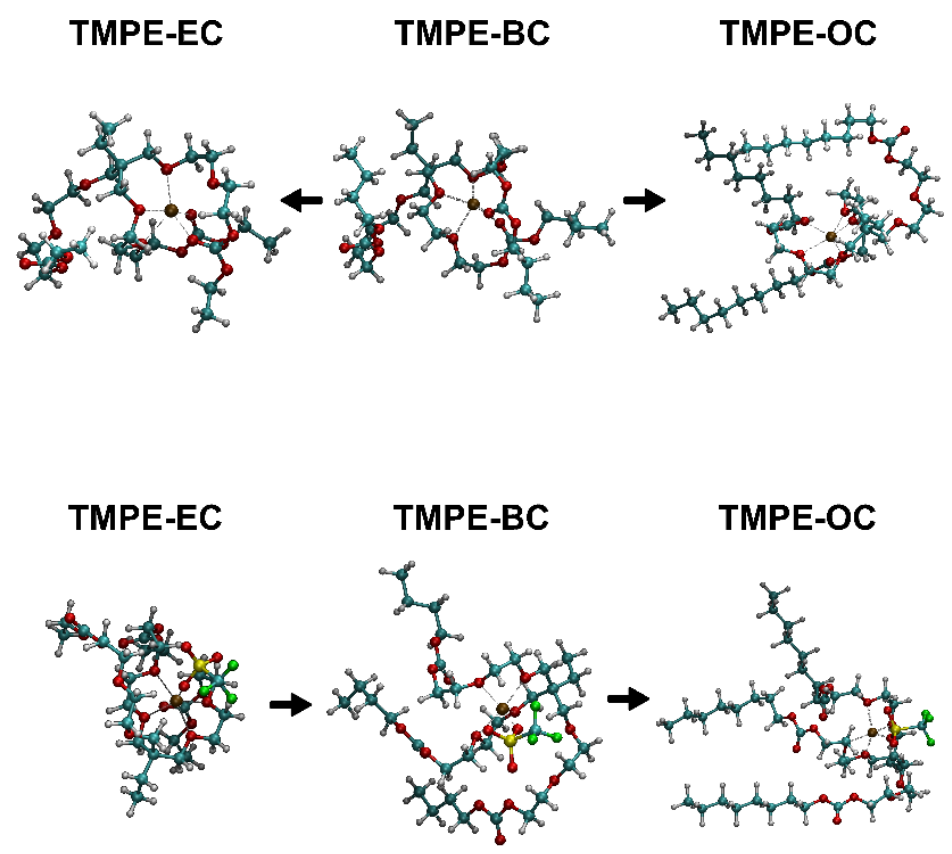

Figure S3. Additional DFT-optimized geometries for TMPE-EC/-BC/-OC complexes. Structures in the top panels show ion transporter $+\mathrm{Li}^{+}$complexes. TMPE-BC and TMPE-OC complexes are built starting from the TMPE-BC complex, which had a stronger-binding minimum energy structure from the MD simulation as compared to TMPE-EC and TMPEOC. The bottom panels show the corresponding results for ion transporter $+\mathrm{Li}^{+}+\mathrm{CF}_{3} \mathrm{SO}_{3}{ }^{-}$ with structures built from the TMPE-EC complex.

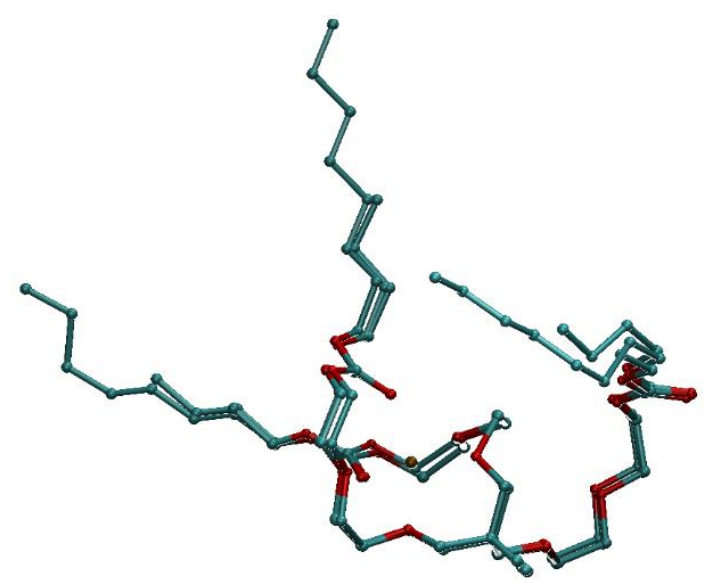

Figure S4. Comparison of the backbones in TMPE-EC/-BC/-OC bound to $\mathrm{Li}^{+}$. For clarity, hydrogens are not shown. The structures are superimposed. 

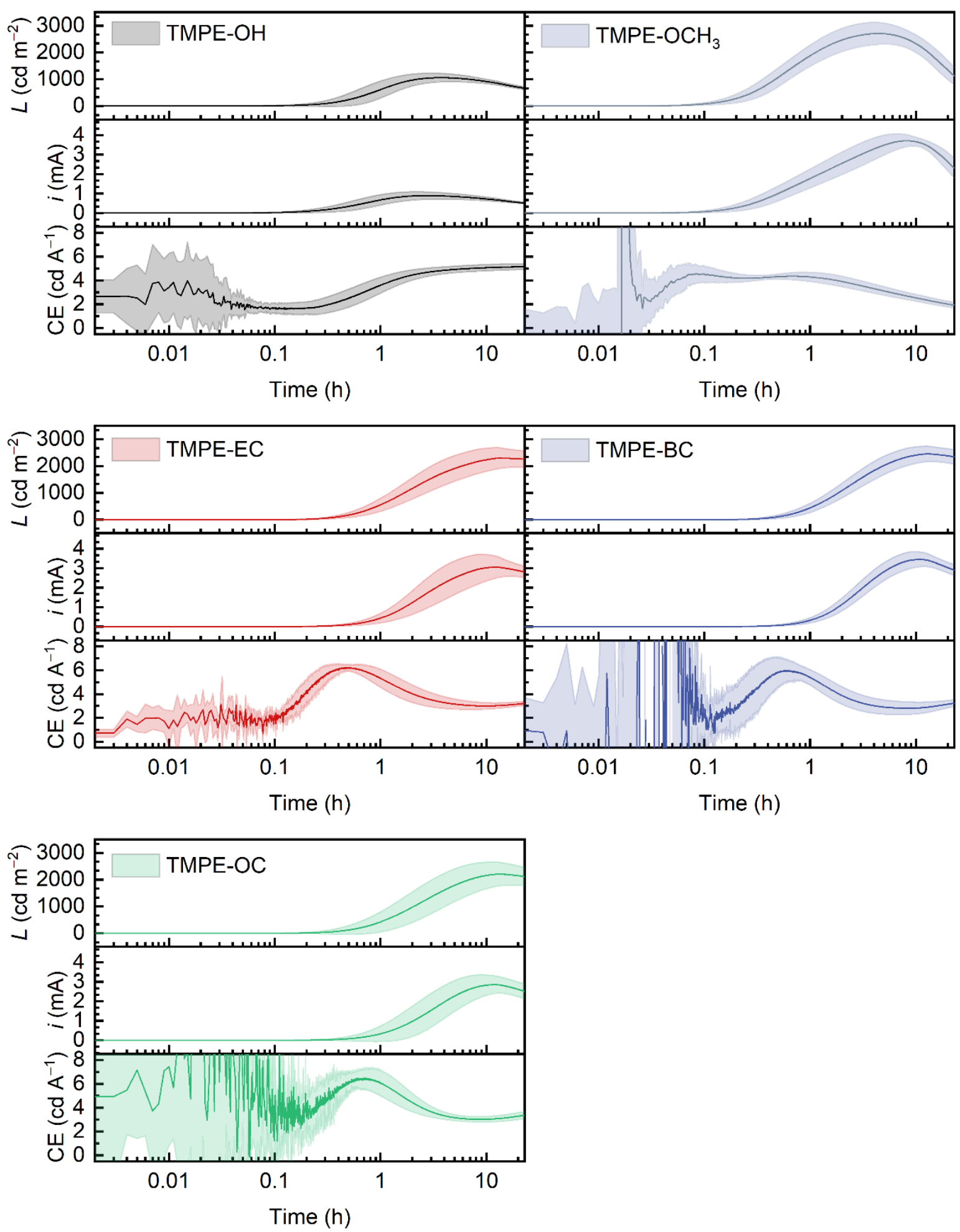

Figure S5. Luminance $(L)$, current $(i)$ and current efficacy (CE) transients for devices made with each of the ion transporters during constant-voltage turn-on at $4 \mathrm{~V}$. Each line represents the average of 5-6 individual devices and the shaded areas represent the standard deviation. 
The noise and sharp peaks in the CE at around $0.1 \mathrm{~h}$ and below should be disregarded as they are artifacts caused by the extremely low current and luminance prior to junction formation

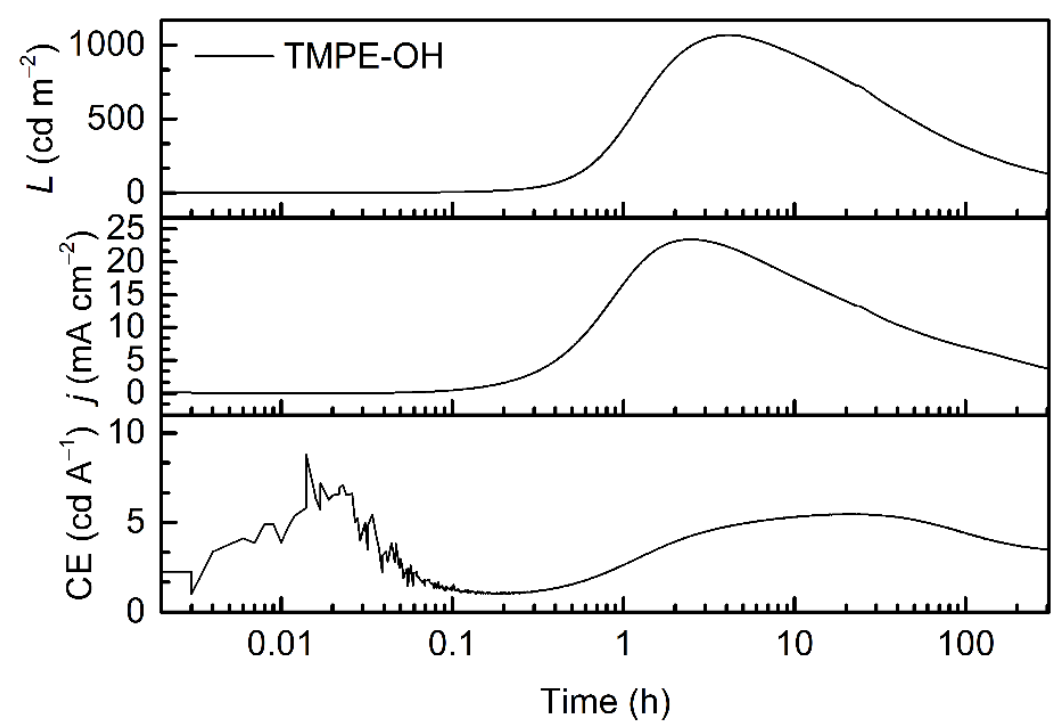

Figure S6. Long-term luminance $(L)$, current density $(j)$ and current efficacy $(C E)$ turn-on transients for a single device made with TMPE-OH as the ion transporter during constantvoltage turn-on at $4 \mathrm{~V}$.

\section{References}

(1) Thordarson, P. Determining association constants from titration experiments in supramolecular chemistry. Chem. Soc. Rev. 2011, 40, 1305-1323.

(2) Howe, E. N.; Bhadbhade, M.; Thordarson, P. Cooperativity and Complexity in the Binding of Anions and Cations to a Tetratopic Ion-Pair Host. J. Am. Chem. Soc. 2014, 136, 7505-7516.

(3) Lanz, T.; Lindh, E. M.; Edman, L. On the asymmetric evolution of the optical properties of a conjugated polymer during electrochemical p- and n-type doping. J. Mater. Chem. C 2017, 5, 4706-4715. 